\title{
Zeitschrift für junge Religionswissenschaft
}

$16 \mid 2021$

Jahresausgabe 2021

\section{Religionswissenschaft, quo vadis?}

Die Entwicklung eines Kleinen Fachs zwischen 2010 und 2030

\section{Ulrike Kollodzeiski}

\section{(2) OpenEdition}

\section{Journals}

Electronic version

URL: https://journals.openedition.org/zjr/1718

DOI: $10.4000 /$ zjr. 1718

ISSN: $1862-5886$

Publisher

Deutsche Vereinigung für Religionswissenschaft

Electronic reference

Ulrike Kollodzeiski, „Religionswissenschaft, quo vadis?", Zeitschrift für junge Religionswissenschaft

[Online], 16 | 2021, Online erschienen am: 06 Oktober 2021, abgerufen am 19 November 2021. URL:

http://journals.openedition.org/zjr/1718; DOI: https://doi.org/10.4000/zjr.1718

This text was automatically generated on 19 novembre 2021 .

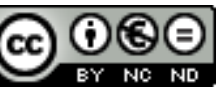

Dieses Werk ist lizenziert unter einer Creative Commons Namensnennung - Nicht-kommerziell - Keine Bearbeitung 3.0 Deutschland Lizenz. 


\title{
Religionswissenschaft, quo vadis?
}

Die Entwicklung eines Kleinen Fachs zwischen 2010 und 2030

\author{
Ulrike Kollodzeiski
}

\section{Einleitung}

1 Der Wissenschaftsrat hat 2010 in einem umfangreichen Bericht die Lage von Theologien und religionsbezogenen Wissenschaften an deutschen Universitäten analysiert und weitreichende Empfehlungen formuliert. In Bezug auf die Religionswissenschaft spricht er sich dafür aus, das Fach weiter auszubauen und durch zusätzliche Professuren zu stärken. Diese Empfehlung knüpft er an die besondere gesellschaftliche Relevanz des Fachs im Angesicht von Prozessen wie Globalisierung und Migration (Wissenschaftsrat 2010, 86-91). Wie hat sich nun die Religionswissenschaft in Deutschland seitdem in den letzten zehn Jahren entwickelt? Wurde den Vorschlägen des Wissenschaftsrats entsprochen und welche Prognose lässt sich daran anschließend für die nächsten zehn Jahre stellen? Diesen Fragen werde ich im Folgenden anhand der Entwicklung der religionswissenschaftlichen Professuren an deutschen Universitäten nachgehen. Dazu gilt es zunächst zu klären, um welche Professuren es dabei geht. Wer zählt überhaupt zur Religionswissenschaft und ist deshalb in die Analyse miteinzubeziehen? Diese Frage ist nicht einfach zu beantworten und verweist bereits auf ein zentrales Problemfeld des Fachs.

Diese Untersuchung orientiert sich vorrangig an den Professuren und kann dadurch nur einen Ausschnitt der Entwicklung des Fachs beleuchten. Keine Beachtung finden hier die Entwicklung von Stellen im Mittelbau (etwa in Bezug auf Entfristung) oder das Verhältnis von Grundfinanzierung und Drittmitteleinwerbungen. Dennoch lassen sich anhand der Professuren als zentrale und wichtigste Strukturstellen bereits entscheidende Tendenzen nachverfolgen. Ihre weitere Entwicklung wird außerdem maßgeblich über die Zukunft des Fachs insgesamt entscheiden. Zudem läuft die Ausbildung und Selektion des wissenschaftlichen Nachwuchses grundsätzlich auf das Ziel Professur zu. Die vorliegende Analyse kann deshalb auch Orientierung bieten, wie 
es um die Chancen steht, dieses Ziel innerhalb der Religionswissenschaften überhaupt zu erreichen.

\section{Wer gehört zur Religionswissenschaft?}

3 Die Religionswissenschaft zählt in Deutschland zu den sog. Kleinen Fächern. Dies suggeriert, dass es sich bei ihr um eine übersichtliche Disziplin handelt, die durch eine begrenzte und klar definierte Anzahl von Professuren vertreten wird. Ein Blick auf die universitäre Landschaft zeigt aber das genaue Gegenteil. Es ist gar nicht so klar, welche Professuren überhaupt zur Religionswissenschaft gehören. Dies betrifft Professuren mit einer explizit religionswissenschaftlichen Denomination, also diejenigen, die bei der Stellenbesetzung in ihrer Ausrichtung durch die universitären Gremien ausdrücklich mit dem Terminus »Religionswissenschaft« versehen wurden. Es betrifft aber ebenso solche Professuren, in deren Denomination nur der Begriff "Religion" auftaucht und die religionsbezogen arbeiten - etwa in der Theologie, Soziologie, Psychologie, Philosophie, Geschichte, Regionalwissenschaft und in vielen weiteren Fächern, die zu einer bestimmten Religion arbeiten. Nur ein kleiner Teil von Letzteren wird auch zur Religionswissenschaft gerechnet, wie ich im Folgenden zeigen werde.

4 Nach der Zählung des Wissenschaftsrats gab es 2009 in Deutschland 28 Universitäten mit religionswissenschaftlichen Professuren. Aktuell sind es noch 27, nachdem der Standort an der TU Dresden 2011 aufgelöst wurde. An 18 Standorten sind die Professuren in theologischen Fakultäten angesiedelt, wobei ein deutlicher Schwerpunkt in der evangelischen Theologie liegt (12 ev., nur 6 kath.). An 16 Standorten sind die Professuren außerhalb der Theologie zu finden (Wissenschaftsrat 2010, 115-117). Das macht deutlich, dass an etlichen Standorten die Religionswissenschaft zugleich an mehreren Fakultäten verortet ist.

5 Im Unterschied zum Wissenschaftsrat verzeichnet das Portal Kleine Fächer aktuell nur 22 Standorte (Kleine Fächer n.d.). Die Deutsche Vereinigung für Religionswissenschaft listet sogar nur 19 verschiedene Universitäten (DVRW n.d.). Die fünf Standorte, die der Wissenschaftsrat mitzählt, die auf der Liste des Portals Kleine Fächer aber fehlen, sind: Freiburg, Konstanz, Köln, Mainz und Trier. Dass der Standort Köln nicht über eine eigene Professur verfügt und der Standort Trier sich derzeit noch im Aufbau befindet mag erklären, warum diese nicht mitgezählt wurden. Warum die anderen drei fehlen, wird nicht ersichtlich. Sie verfügen jeweils über mindestens eine Professur mit einer religionswissenschaftlichen Denomination, die an den drei Standorten an unterschiedlichen Fakultäten angesiedelt ist. Auch die DVRW listet diese fünf Standorte nicht. Bei ihr sind außerdem nicht verzeichnet: HU Berlin, Erlangen-Nürnberg und Halle-Wittenberg. Diese sind alle in theologischen Fachbereichen angesiedelt. An letzterem Standort etwa wird die "Professur für Religionswissenschaft und interkulturelle Theologie« an der evangelisch-theologischen Fakultät durch Daniel Cyranka vertreten, der sogar selbst Mitglied der DVRW ist (Uni Halle n.d.). Keine der Zählungen listet den Standort an der evangelisch-theologischen Fakultät der Universität Rostock mit der »Professur für Religionsgeschichte - Religion und Gesellschaft«, bis vor kurzem besetzt mit Klaus Hock (Kleine Fächer n.d.).

6 Auch darüber, welche Professuren an einem Standort zur Religionswissenschaft gezählt werden, sind sich die Erhebungen nicht einig. Dies zeigt das Beispiel Münster. Das Portal Kleine Fächer listet hier nur die »Professur für Religionswissenschaft« an der katholisch- 
theologischen Fakultät (aktuell Astrid Reuter) (Kleine Fächer n.d.). Der Wissenschaftsrat und die DVRW dagegen zählen auch die »Professur für Religionswissenschaft und Interkulturelle Theologie« an der evangelisch-theologischen Fakultät (Perry Schmidt-Leukel) dazu (Wissenschaftsrat 2010, 116). Keiner von ihnen bezieht aber die "Professur für Religionssoziologie« am Fachbereich für Erziehungsund Sozialwissenschaften (Detlef Pollack) in ihre Rechnung mit ein.

In Bezug auf den letztgenannten Aspekt zeigt sich wiederum ein anderes Bild bei einem Blick auf den Standort Göttingen. Hier arbeiten drei Professuren betont interdisziplinär in einem Institut zusammen und verorten sich alle in der Religionswissenschaft. Vertreten sind die evangelisch-theologische Fakultät (Andreas Grünschloß), die philosophische Fakultät (Ilinca Tanaseanu-Döbler) und die sozialwissenschaftliche Fakultät (Alexander-Kenneth Nagel). Sie werden auch alle durch das Portal Kleine Fächer gelistet (Kleine Fächer n.d.). ${ }^{1}$

Bei den genannten Kartierungen sind zwei Tendenzen auffällig:

1. Insgesamt werden nur solche Standorte bzw. Professuren als religionswissenschaftlich gezählt, die auch explizit in ein religionswissenschaftliches Institut und/oder einen entsprechenden Studiengang eingebunden sind. Es bedarf also einer doppelten institutionellen Positionierung. Die Denomination einer Professur reicht nicht aus, sondern eine weitere Markierung als religionswissenschaftlich ist notwendig, um als solche wahrgenommen zu werden.

2. Diejenigen Standorte bzw. Professuren, die trotz einer ausdrücklich religionswissenschaftlichen Denomination nicht mitgezählt werden, sind vor allem solche, die an theologischen Fakultäten angesiedelt sind. Offen bleibt dabei aber, warum ausgerechnet bestimmte Professuren nicht dazugerechnet werden - im Gegensatz zu anderen mit der gleichen Denomination und Einbettung.

9 Der Wissenschaftsrat problematisiert in seinem Bericht, dass der Platz der Religionswissenschaft im Fächerspektrum bisher noch unklar geblieben sei (Wissenschaftsrat 2010, 86). Außerdem zählt er zwar die religionswissenschaftlichen Standorte getrennt danach, ob sie innerhalb oder außerhalb der Theologie angesiedelt sind. Er verzichtet aber auf eine Erhebung der genauen Anzahl von Professuren. Hervorgehoben wird stattdessen, dass die Lage des Fachs unübersichtlich sei, da an einigen Standorten »die Religionswissenschaft >doppelt» vertreten« ist (Wissenschaftsrat 2010, 48).

10 Der kurze Vergleich dieser drei Kartierungen der Religionswissenschaft weist damit schon auf eine Eigenschaft des Fachs hin, die auch an anderen Stellen immer wieder deutlich wird: Das Fach definiert sich über seinen Gegenstand Religion, den es auf methodisch-theoretisch vielfältige Weise behandelt. Sowohl seinen Gegenstand als auch seine Methoden und Theorien teilt es aber mit vielen anderen Disziplinen. Zu den meisten verwandten Fächern ist das Verhältnis schlicht nicht geklärt und wird auch kaum thematisiert. In Bezug auf die Theologie dagegen bildet es einen stetigen Diskussionsgegenstand. Grund dafür ist wohl nicht zuletzt, dass an jedem zweiten Standort eine religionswissenschaftliche Professur an einer theologischen Fakultät angesiedelt ist. Diese machen also einen wesentlichen Bestandteil der Religionswissenschaft in Deutschland aus. 


\section{Die Entwicklung der Religionswissenschaft seit 2010}

11 Der Wissenschaftsrat hat in seinem Bericht 2010 in der ungeklärten Lage der Religionswissenschaft auch ein strukturelles Problem erkannt: An den meisten Standorten sei die Religionswissenschaft mit zu wenigen Professuren ausgestattet, um tatsächlich als eigenständiges Fach auftreten zu können. Sie steht entweder im Dienst eines größeren Fachs und bildet nur eine Hilfswissenschaft, oder sie ist zu sehr auf den Import aus anderen Fächern angewiesen, um ihr eigenes Profil umfassend durchsetzen zu können. Daher hat der Wissenschaftsrat empfohlen,

"dass an mehreren Standorten in Deutschland religionswissenschaftliche Institute, d.h. auf Dauer gestellte größere Lehr- und Forschungseinheiten mit einer Mindestzahl von vier Professuren, eingerichtet werden. Einheiten einer solchen Größe können dann auch eigenständige religionswissenschaftliche Studiengänge auf Bachelor- und auf Masterniveau anbieten und verfügen grundsätzlich über die Voraussetzungen, um systematische und komparative Forschung betreiben $\mathrm{zu}$ können. Dabei sollten die inhaltlichen und methodischen Schwerpunktsetzungen dieser religionswissenschaftlichen Institute durchaus unterschiedlich sein.« (Wissenschaftsrat 2010, 87)

Eigentlich böte das Portal Kleine Fächer einen Überblick, welche Standorte und Professuren in der Religionswissenschaft in den vergangenen Jahren dazugekommen oder weggefallen sind. Da aber die Angaben wie eben dargestellt zum Teil problematisch sind, sind diese Daten leider nur bedingt aussagekräftig. Sie können aber zumindest Tendenzen in der Entwicklung aufzeigen. Für 2010 zählte das Portal insgesamt 37 Professuren. 2021 sind es gemäß dieser Zählung insgesamt 46 Professuren (Kleine Fächer n.d.). Das bedeutet einen deutlichen Zuwachs um etwa ein Viertel. An jedem zweiten Standort kam innerhalb der letzten zehn Jahre eine neue religionswissenschaftliche Professur dazu. An acht Standorten ist die Religionswissenschaft inzwischen mit drei oder mehr Professuren vertreten (Bayreuth, Bochum, Bremen, Erfurt, Göttingen, Heidelberg, Leipzig und München). Es sind aber nicht nur Professuren hinzugekommen, in Erfurt und an der FU Berlin ist jeweils auch eine Professur weggefallen. Wie bereits erwähnt, entfiel auch der Standort Dresden mit einer Professur, dieser ist aber nicht Teil der Statistik des Portals. Es gilt hier außerdem darauf hinzuweisen, dass jüngst viele Kleine Fächer, die ebenfalls religionsbezogen arbeiten, von Standortschließungen betroffen sind. Ein erschreckendes Beispiel hierfür ist die Indologie: Das Portal Kleine Fächer listet für 2010 noch insgesamt 18 Professuren an 15 universitären Standorten. Aktuell verzeichnet es nur noch 14,5 Professuren an 11 Standorten (Kleine Fächer n.d.). Dabei sind bevorstehende Schließungen noch nicht mit eingerechnet, wie in Göttingen und Köln, die bereits beschlossen wurden, sowie in Halle, wo noch dagegen gekämpft wird.

Die Religionswissenschaft hat in den letzten zehn Jahren einen erheblichen personalen Wechsel durchgemacht. Es wurden nicht nur neue Professuren geschaffen und erstmals besetzt, auch viele schon länger bestehende Professuren wurden neu besetzt. An den vom Wissenschaftsrat gelisteten Standorten sind aktuell ca. 28 Professoren und Professorinnen beschäftigt, die innerhalb der letzten zehn Jahre auf diese Positionen berufen wurden. ${ }^{2}$ Das entspricht mehr als der Hälfte aller religionswissenschaftlichen Professuren in Deutschland. Eine erfreuliche Entwicklung lässt sich dabei in Bezug auf das Geschlechterverhältnis feststellen: Von den 28 Berufenen waren jeweils 14 weiblich und 14 männlich. Diese Neubesetzungen fanden zu zwei Dritteln an Standorten 
außerhalb der Theologie statt: 19 außerhalb gegenüber 9 innerhalb von theologischen Fakultäten. Das heißt, dass die Religionswissenschaft außerhalb der Theologie stärker gewachsen ist als innerhalb dieser.

Bei den Neuausschreibungen außerhalb von theologischen Fakultäten zeigt sich ein buntes Bild sowohl in Bezug auf die Disziplin als auch den Gegenstandbereich der Denominationen. Es finden sich soziologische, religionsgeschichtliche, kulturwissenschaftliche, medienwissenschaftliche und religionskritische Ausrichtungen. Schwerpunkte wurden sowohl gegenwartsbezogen als auch historisch gesetzt, ebenso in Bezug auf die Regionen Asien und Afrika sowie auf einzelne Religionen wie Judentum, Islam, Christentum aber auch Zoroastrismus. An vielen Standorten wurde so das Spektrum um bestehende Professuren erweitert, die schlicht den Titel »Allgemeine Religionswissenschaft« tragen und bisher oftmals alles abdecken mussten. Den Empfehlungen des Wissenschaftsrats wurde also vielfach entsprochen. Dieses neue und vielfältigere Bild weist jedoch immer noch weiße Flecken auf, etwa im Bereich der Psychologie, Ethnologie und der Philosophie, aber auch große Teile des globalen Südens treten bisher überhaupt nicht in Erscheinung. Zwar sind hier asiatische Regionen traditionell stark vertreten, andere Kontinente dagegen wie etwa Mittel- und Südamerika oder Australien fehlen gänzlich. Die Religionswissenschaft ist in Deutschland gemessen an den Denominationen der Professuren stark fokussiert auf Europa und Asien, die es geistes- und kulturwissenschaftlich erforscht. Diese Herangehensweise wurde in den letzten Jahren allerdings stärker um soziologisch ausgerichtete Professuren ergänzt.

15 In den theologischen Fakultäten blieb die Religionswissenschaft häufig mit nur einer Professur vertreten, deren religionswissenschaftliche Denomination in der Regel kombiniert ist mit »interkultureller Theologie« oder »Missionswissenschaft» (in der evangelischen Theologie) oder »Fundamentaltheologie» (in der katholischen Theologie). Aber auch hier wurden an einigen Standorten jüngst neue Wege eingeschlagen - in unterschiedliche Richtungen. In Münster erfolgte dieses Jahr die Ausschreibung einer zusätzlichen Juniorprofessur für Religionswissenschaft innerhalb der evangelisch-theologischen Fakultät ohne Konfessionsbindung. Dagegen entschied sich der Fachbereich katholische Theologie in Frankfurt a.M. 2018, die dortige Professur neu als »Religionstheologie und Religionswissenschaft« auszuschreiben und damit wieder stärker theologisch auszurichten.

16 Aufgabe dieser einzelnen Professuren an theologischen Fakultäten ist es häufig, religionswissenschaftliche Kompetenzen in die Pfarr- bzw. Priester- und Lehramtsausbildung einzubringen. Sie haben so die Möglichkeit, einen wichtigen gesellschaftlichen Beitrag zu leisten, indem sie diese Kompetenzen an zukünftige Multiplikatoren vermitteln. Damit kommt ihnen in der Religionswissenschaft eine besondere Relevanz zu, denn die Professuren außerhalb der theologischen Fakultäten sind meist nicht in die Lehramtsausbildung eingebunden. Das Schulfach Religion wird in den meisten Bundesländern entweder von den Religionsgemeinschaften - allen voran der evangelischen und katholischen Kirche - verantwortet oder alternativ als Teilthema im Ethikunterricht abgedeckt. Hiervon abweichende, je eigene Modelle finden in Brandenburg, Bremen, Hamburg und Niedersachsen Anwendung, wobei der »Religionsunterricht für alle« in Hamburg von Religionsgemeinschaften verantwortet wird und Niedersachsen das Alternativfach »Werte und Normen« in Kooperation mit dem Fachverband Ethik anbietet, der wiederum eng mit dem Humanistischen Verband 
verflochten ist. Einen dezidiert staatlichen Religionskundeunterricht gibt es nur in Brandenburg und Bremen. (Zum Einstieg in die komplexe Debatte um den Religionsunterricht siehe: Schröder 2020; Kenngott, Englert und Knauth 2015).

In jedem zweiten Fall findet sich neben der religionswissenschaftlichen Professur in der Theologie an derselben Universität noch eine oder zwei weitere solcher Professuren in anderen Fakultäten, die gemeinsam einen religionswissenschaftlichen Studiengang betreuen. Wie das Beispiel Göttingen oben bereits gezeigt hat, können dabei auch religionswissenschaftliche Professuren an theologischen Fakultäten wesentlich zum Aufbau eines größeren, Disziplinen übergreifenden Standorts beitragen. Dieses Beispiel stellt damit aber auch eine Empfehlung des Wissenschaftsrats in Frage: Für eine bessere Identifizierbarkeit und eigenständigere Entwicklung des Fachs hatte sich dieser dafür ausgesprochen, dass religionswissenschaftliche Professuren an theologischen Fakultäten auf die Bezeichnung »Religionswissenschaft» verzichten sollten, sofern sie vorrangig als Hilfswissenschaften der Theologie auftreten. Sie sollten dann nur noch als »Missionswissenschaft« bzw. »interkulturelle Theologie» oder als »Fundamentaltheologie« bezeichnet werden (Wissenschaftsrat 2010, 88f.). Der Wissenschaftsrat hat außerdem empfohlen,

»die entsprechenden Professuren in religionswissenschaftliche Institute außerhalb theologischer Fakultäten $\mathrm{zu}$ verlagern. Eine solche Verlagerung der Religionswissenschaft aus der Theologie heraus ist aufgrund der staatskirchenrechtlichen Bindungen mit zusätzlichen Kosten verbunden. Sie ist jedoch entscheidend für die Weiterentwicklung des Faches. Ziel sollte es sein, getrennte religionswissenschaftliche und theologische Studiengänge zu schaffen. Dabei kann eine Kooperation beider Bereiche über das Instrument der Doppelprofessur sichergestellt werden.« (Wissenschaftsrat 2010, 88)

Dieser Empfehlung wurde in den letzten Jahren kaum gefolgt. Weder haben die theologischen Fakultäten die religionswissenschaftlichen Professuren an andere Fakultäten abgegeben, noch haben sie vermehrt in deren Denominationen auf die Bezeichnung "Religionswissenschaft" verzichtet. Hannover ist eines der wenigen Beispiele für eine klarere Trennung von Theologie und Religionswissenschaft. Hier hatten seit 2009 beide in einem Institut innerhalb der Philosophischen Fakultät zusammengearbeitet. 2019 trennten sie sich jedoch und eigenständige Institute für Religionswissenschaft einerseits und Theologie andererseits wurden geschaffen (Uni Hannover n.d.). Auch das "Instrument der Doppelprofessur" fand bisher nur im Einzelfall Anwendung, etwa bei Michael Bergunder in Heidelberg, dessen Professur an der evangelischen Fakultät verortet aber auch an der philosophischen Fakultät im Institut für Religionswissenschaft kooptiert ist (Uni Heidelberg n.d.).

Diese Strategie der stärkeren institutionellen Abgrenzung von Theologie und Religionswissenschaft hat sich in den letzten Jahren nicht durchsetzen können. Im besten Fall entfiel die Konfessionsbindung bei einer Neubesetzung, aber die Professur verblieb an der theologischen Fakultät. Das Beispiel Göttingen zeigt außerdem, dass nicht Abgrenzung, sondern stärkere interdisziplinäre Zusammenarbeit eine alternative Strategie sein kann. Natürlich muss dann eine Fakultät und Kirche bereit sein, einer Professur mehr Unabhängigkeit einzuräumen. Aber auch die Akzeptanz von Seiten der Religionswissenschaft für die Kollegen und Kolleginnen an theologischen Fakultäten muss dazu wachsen. Es darf nicht in Frage stehen, ob sie überhaupt zur Religionswissenschaft gehören. 
20 An manchen Standorten wurden neue, zusätzliche religionswissenschaftliche Professuren zunächst als Juniorprofessuren ausgeschrieben, die aber mit einer tenuretrack-Option ausgestattet waren und damit auch langfristig zur Stärkung der Standorte beitragen, so etwa in Bayreuth, Göttingen und jüngst Münster. An dieser Stelle bleibt die Frage nach der Finanzierung solcher zusätzlichen Professuren ungeklärt. Handelt es sich wirklich um neu geschaffene Professuren oder wurde umverteilt? An einigen Standorten wurden Juniorprofessuren auch ohne tenure-track ausgeschrieben, so etwa 2020 in Leipzig oder in Hamburg. Letzterer Standort lässt zugleich die Komplexität der Konstruktionen und Umstrukturierungsprozesse erahnen: Das Institut für Missions-, Ökumene- und Religionswissenschaft an der evangelischen Fakultät war zunächst mit zwei Professuren ausgestattet, der Professur »Globales Christentum, Missions- und Ökumenewissenschaften « und der Professur »Missions-, Ökumene- und Religionswissenschaft«. Erstgenannte wurde nach der Emeritierung von Theodor Ahrens 2005 nicht neu besetzt, dafür kam 2018 zur W3-Professur »Missions-, Ökumeneund Religionswissenschaft « (Ulrich Dehn bis 2020, vertreten derzeit durch Ulrike Auga) eine Juniorprofessur ohne tenure-track mit derselben Denomination (Giovanni Maltese) hinzu (Uni Hamburg n.d.).

21 Zwar wurden jüngst vor allem Juniorprofessuren ausgeschrieben, aber es lässt sich in der Religionswissenschaft bisher nicht die Tendenz feststellen, dass diese flächendeckend als preiswertere Alternative W2- und W3-Professuren ersetzen würden. Sie dienen vielmehr dem langfristigen Aufbau (bzw. der Umstrukturierung) von Standorten und schaffen für den wissenschaftlichen Nachwuchs die Möglichkeit für einen früheren Einstieg in eine dauerhafte Laufbahn auf einer Professur. Die Etablierung neuer Professuren über das Mittel der Juniorprofessur hat freilich die Folge, dass diese Stellen nun auf lange Zeit besetzt sind. Auch aktuell profitiert nur ein begrenzter Teil des wissenschaftlichen Nachwuchses vom Modell der Juniorprofessur, nämlich nur diejenigen, die zwar promoviert, aber noch nicht habilitiert sind, die zwar schon einen Lebenslauf vorzuweisen haben, der sie als berufungsfähig ausweist, deren Promotion aber noch nicht zu lange zurückliegen darf. Das biografische Zeitfenster, das sich so für eine aussichtsreiche Bewerbung auftut, ist sehr kurz. Hinzu kommen weitere einschränkende Kriterien wie etwa ein bestimmter Schwerpunkt, der gesucht wird, und unter Umständen die Zugehörigkeit zu einer bestimmten Konfession.

\section{Prognose für die Religionswissenschaft bis 2030}

Wird sich der positive Trend der letzten Jahre fortsetzen und weitere Professuren geschaffen werden, hat das Fach inzwischen ein Plateau erreicht oder werden gar religionswissenschaftliche Professuren wegfallen?

Der Wissenschaftsrat hat 2010 seine Empfehlung zum Ausbau religionswissenschaftlicher Standorte an die gesellschaftliche Relevanz des Fachs geknüpft. Die »Rückkehr der Religion«, Prozesse von Globalisierung und Migration und damit einhergehende Konfliktpotentiale habe die Religionswissenschaft $\mathrm{zu}$ einem gefragten Fach werden lassen (Wissenschaftsrat 2010, 86). Zwar teile das Fach sowohl seinen Gegenstand als auch seine methodisch-theoretische Herangehensweise mit vielen anderen Fächer, es verfüge aber auch über eine spezifische Kompetenz, die von großer gesellschaftlicher Relevanz sei. Diese Kompetenz bestehe 
»in ihrem systematischen Anspruch sowie in ihrer komparativen Perspektive, mit der sie empirisch und theoretisch-systematisch religiöse Phänomene innerhalb und außerhalb moderner westlicher Gesellschaften erforscht. Hierin und in der darauf beruhenden Syntheseleistung liegt wissenschaftstheoretisch die Eigenständigkeit der Disziplin begründet.« (Wissenschaftsrat 2010, 87)

Das Interesse an der Religionswissenschaft hatte sich damals u.a. in hohen Studierendenzahlen niedergeschlagen, die zum Teil sogar die Kapazitäten einzelner Standorte überstiegen. Dagegen wurden beim "Professorium« der Jahrestagung der DVRW in Hannover 2019 sinkende Studierendenzahlen und eine zum Teil mangelnde Auslastung vor allem der Masterstudiengänge beklagt. Folgt man jedoch den Angaben des Statistischen Bundesamts, dann sind die Zahlen zwar in den letzten Jahren leicht rückläufig, bewegen sich aber noch immer auf einem relativ hohen Niveau. Zum Vergleich: Im WiSe 2010/11 waren insgesamt 4.750 Studierende in einem religionswissenschaftlichen Studiengang (1., 2. und 3. Fach zusammen) eingeschrieben (DeStatis 2010/11, 303), im WiSe 2015/16 waren es insgesamt 5.548 (DeStatis 2015/16, 319), und im WiSe 2019/20 insgesamt 4.968 Studierende (DeStatis 2019/20, 315). Als Bachelor-Fach ist die Religionswissenschaft nach wie vor beliebt - vor allem als zweites Fach. Das Verhältnis von Erst- zu Zweitfach liegt hier über die Jahre relativ stabil bei 2:3. Besorgniserregend dagegen ist die Entwicklung der Studierendenzahlen über den Verlauf der Fachsemester. Sie nimmt schon zwischen dem ersten und zweiten Studienjahr um ca. ein Drittel ab, das heißt, ca. ein Drittel aller Studierenden der Religionswissenschaft verlässt das Fach nach dem ersten Studienjahr wieder. Dieses Problem besteht bereits seit Jahren. Es deckt sich auch mit dem Befund, dass an vielen Standorten die Einschreibezahlen in den religionswissenschaftlichen BAStudiengängen zwar gut sind - nicht aber die Abschlusszahlen. Die Religionswissenschaft teilt dieses Problem mit vielen anderen Geistes- und Kulturwissenschaften. Es trifft sie aber anders als etwa die Philosophie und Geschichte, die etwa 2019/20 über insgesamt 41.571 (Philosophie) und sogar 68.110 (Geschichte) Studierende verfügten. Auch sie verlieren zwischen dem ersten und zweiten Studienjahr etwa ein Viertel bis ein Drittel ihrer Studierenden (DeStatis 2019/20, 315f.). Allerdings fällt dieser Verlust aufgrund der viel höheren Studierendenzahlen weniger stark ins Gewicht als in der Religionswissenschaft, wo er zu einer Unterauslastung der höheren Fachsemester führt.

Zudem ist der Anteil der Masterstudierenden relativ gering, sie machen nur ca. $10 \%$ aller Studierenden im Fach Religionswissenschaft aus. Allerdings ist hier die Zahl zuletzt etwas gestiegen. Im WiSe 2019/2020 lag die Zahl aller Studierenden, die einen religionswissenschaftlichen Masterabschluss anstreben, bei 670 (DeStatis 2019/20, 340). Damit wird schnell klar, dass sich nicht an jedem religionswissenschaftlichen Standort ein Bachelor- und Masterstudiengang halten lässt. Bezogen auf die Studierendenzahlen wäre es rein rechnerisch ausreichend, wenn an knapp zehn, also ungefähr jedem dritten Standort ein religionswissenschaftlicher Master angeboten würde. Momentan sind es laut Hochschulkompass zwölf Standorte, die einen Master »Religionswissenschaft « anbieten. Zusätzlich gibt es aber ungefähr noch einmal so viele eng verwandte, oft interdisziplinär angelegte Masterprogramme wie etwa »Religion und Kultur« an der Humboldt Universität zu Berlin (Hochschulkompass n.d.).

Die Studierendenzahlen in den religionswissenschaftlichen Studiengängen sind damit über die Jahre als relativ stabil zu beschreiben. Es gibt einen leichten, aber stetigen Abwärtstrend in den Bachelorstudiengängen und (bei einer sehr optimistischen 
Betrachtungsweise) einen leichten Aufwärtstrend in den Masterstudiengängen. Interessant wird sein, wie sich diese Zahlen nun während und nach der Pandemie entwickeln. In jedem Fall haben sich die etwa gleichbleibenden Studierendenzahlen aber in den letzten Jahren nicht proportional zu denen der Professuren entwickelt, deren Zahl um ca. ein Viertel gestiegen ist. Es steht deshalb kaum zu erwarten, dass unter den derzeitigen Umständen in den nächsten Jahren viele weitere religionswissenschaftliche Professuren geschaffen werden.

Der wissenschaftliche Nachwuchs von heute kann deshalb kaum damit rechnen, dass in den nächsten zehn Jahren ebenso viele Professuren ausgeschrieben werden wie in den letzten zehn. Wahrscheinlich werden es gerade einmal halb so viele sein - nämlich nur die, die aufgrund von Pensionierungen neu besetzt werden müssen. Gemessen am Alter gilt es bis $2030 \mathrm{ca} .13$ Professuren $^{3}$ neu zu besetzen. Einige wenige sind darüber hinaus gerade vakant oder noch im Aufbau begriffen. Das bedeutet, dass in den nächsten Jahren durchschnittlich jeweils ein bis maximal zwei Professuren pro Jahr ausgeschrieben werden. Von diesen ist rund die Hälfte in evangelisch-theologischen Fakultäten. Deren Ausschreibungen werden also maßgeblich darüber mitentscheiden, wie sich die Religionswissenschaft in den nächsten zehn Jahren weiterentwickelt. Sollten sie auf eine Konfessionsbindung setzen, reduziert sich die Anzahl der neu zu besetzenden Professuren für den nicht-evangelischen Nachwuchs auf ein halbes Dutzend. Die Frage ist auch, ob diese Professuren als Juniorprofessuren auf den Markt kommen - mit den oben genannten Folgen für das kurze biografische Zeitfenster, in dem es überhaupt möglich ist, auf eine solche Professur zu kommen.

Um die Brisanz dessen weiter deutlich zu machen: Diesen wenigen Professuren stehen derzeit so viele Promovierte und Promovierende gegenüber wie noch nie in der Geschichte des Faches. Allein für 2019 verzeichnete das Statistische Bundesamt 263 Promovierende nur im Fach Religionswissenschaft. Der Zuwachs an Professuren, Drittmittelprojekten mit integrierten Promotionen und der Ausbau von Graduiertenkollegs und Stipendienprogrammen haben hierfür in den letzten Jahren die Rahmenbedingungen geschaffen. Die Möglichkeiten zu promovieren wurden damit entschieden verbessert. Allerdings korrespondiert der Zuwachs an Promotionsmöglichkeiten nur sehr bedingt mit einem Zuwachs anschlussfähiger PostDoc-Stellen. $\mathrm{Zu}$ den Promovierenden in der Religionswissenschaft kommen außerdem diejenigen aus angrenzenden Fächern wie etwa der Geschichte, Soziologie oder den Theologien hinzu. Allein in der evangelischen Theologie waren es im selben Jahr 1.080 Promovierende, vertreten natürlich mit einem breiten Fächerspektrum innerhalb der Theologie (DeStatis 2020, 22). Auch sie können sich aber ggf. auf religionswissenschaftliche Lehrstühle bewerben, sofern deren Ausschreibung allein über inhaltliche Faktoren eingegrenzt und nicht explizit an eine religionswissenschaftliche Promotion gebunden ist. Eine solche Bindung stellt für die Neubesetzung derjenigen Professuren eine besondere Herausforderung dar, die mit einem Schwerpunkt auf einen bestimmten Gegenstandsbereich oder Methodik ausgerichtet sind, da es allein innerhalb der Religionswissenschaft oft nur einen kleinen Kreis gibt, der gerade über die notwendigen Qualifikationen verfügt. Entscheidet man sich aber gegen das Kriterium der religionswissenschaftlichen Promotion, dann stellt sich für den Nachwuchs einmal mehr die Frage, inwiefern es sich für ihn überhaupt lohnt, auf eine solche Promotion zu setzen. Der religionswissenschaftliche Nachwuchs muss dann in den nächsten zehn Jahren um die 
wenigen Professuren auch noch mit dem Nachwuchs aus anderen, nicht selten größeren Fächern konkurrieren.

Diese Lage wäre weniger prekär, wenn es mehr konkrete Alternativen für den Nachwuchs gäbe. Aber eine religionswissenschaftliche Dauerstelle an einer deutschen Universität zu ergattern ist ungefähr so wahrscheinlich, wie auf eine Professur zu kommen, weil die meisten Universitäten Dauerstellen nur dann einrichten, wenn sie eine routinisierbare Daueraufgabe erfüllen (meist Sprachausbildung oder Koordination). Das bedeutet, dass für die meisten nach Ausschöpfung der durch das Wissenschaftszeitgesetz festgelegten Jahre unweigerlich Schluss ist. Oder man hangelt sich von Projekt zu Projekt, wobei kaum noch nachvollziehbar ist, inwiefern auch Stellen in Drittmittelprojekten unter die zwölf-Jahre-Regel fallen. Es bleiben einige Stellen an außeruniversitären Forschungseinrichtungen. Der Weg für die allermeisten Promovierenden der Religionswissenschaft führt also früher oder später aus der Universität heraus. Religionswissenschaftliche Kompetenzen sind dort zwar oft nachgefragt, aber meist nur als eine zusätzliche Kompetenz innerhalb eines anderen Berufsfelds. Das macht die Religionswissenschaft zwar als zweites Bachelorfach attraktiv, als alleiniges Master- oder Promotionsfach wirft sie hingegen erhebliche Probleme auf, von einer Habilitation ganz zu schweigen.

30 Es ist an dieser Stelle auch nicht hilfreich, dass sich die Religionswissenschaft in gesellschaftlichen und politischen Fragen meist auf einen Beobachterstatus zurückzieht und keine Positionierungen und Handlungsempfehlungen wagt. Dies schneidet den Transfer von religionswissenschaftlichem Wissen in außeruniversitäre Diskurse ab und lässt die Religionswissenschaft gesellschaftlich irrelevant werden. Religionsbezogene Fragen werden an andere Disziplinen gerichtet und durch Theologie, Soziologie oder Geschichte beantwortet. Der Wissenschaftsrat hat seine Empfehlung für den Aufbau religionswissenschaftlicher Professuren aber an die große gesellschaftliche Relevanz einer spezifisch religionswissenschaftlichen Kompetenz gebunden. Es stellt sich die Frage, wo und wie diese Kompetenz in den letzten zehn Jahren durch die Religionswissenschaft in die Gesellschaft eingebracht werden konnte.

Der Wissenschaftsrat hat 2010 auf Globalisierung und Migration und deren Konfliktpotentiale hingewiesen, welche die Religionswissenschaft zu einem gefragten Fach haben werden lassen. Diese Prozesse haben sich in den letzten zehn Jahren deutlich verschärft. Von einem stärkeren Auftreten der Religionswissenschaft in öffentlichen Debatten, in beratenden oder gar entscheidungstragenden Funktionen kann aber bisher nur in Einzelfällen die Rede sein. Michael Blume ist ein solches Beispiel. Das Fach hat zwar von diesen Prozessen profitiert, indem religionswissenschaftliche Professuren geschaffen wurden, es hat seinen Output in die Gesellschaft aber insgesamt nicht entsprechend erhöht. Einen aufrüttelnden Beitrag hatte bereits Christian Röther anlässlich der DVRW-Jahrestagung 2017 in Marburg verfasst, wo man Bezüge zu aktuellen Themen wie dem Lutherjubiläum oder dem »Islamischen Staat« vergebens suchte (Röther 2017). Inzwischen hat sich zwar etwas getan, auch weil der wissenschaftliche Nachwuchs in seinen Promotionen verstärkt aktuell gesellschaftliche Fragen in den Blick nimmt. Auch Masterprogramme werden derzeit vermehrt auf aktuelle Themen ausgerichtet, oft interdisziplinär angelegt wie etwa der MA »Transkulturelle Studien« in Bremen. An vielen Stellen werden zudem neue Medien wie Blogs und Podcast genutzt, um einen Transfer von religionswissenschaftlichem Wissen in eine breitere öffentlichkeit zu erreichen. 
Insgesamt tritt das Fach aber über den universitären Rahmen hinaus immer noch sehr wenig in Erscheinung. Zu oft ist es gerade nicht die Religionswissenschaft, sondern sind es andere Fächer, die zu religionsbezogenen Themen gehört werden. Dies wird kaum dazu führen, dass in Zukunft weitere Professuren geschaffen werden. Es führt auch nicht dazu, dass außerhalb von Universitäten Religionswissenschaftler und Religionswissenschaftlerinnen gefragte Bewerber und Bewerberinnen sind. Für den wissenschaftlichen Nachwuchs des Fachs ergibt sich daraus sogar ein doppeltes Dilemma.

\section{Fazit}

Die aktuellen gesellschaftlichen Entwicklungen begünstigen eigentlich einen weiteren Ausbau der Religionswissenschaft. Allerdings muss das Fach diese Potentiale besser nutzen, sonst wird sich der positive Trend der letzten Jahre in sein Gegenteil verkehren und andere Fächer davon profitieren. Die Universitäten sind den Empfehlungen des Wissenschaftsrats gefolgt und in Vorleistung gegangen, indem sie der Religionswissenschaft mehr Professuren eingeräumt haben. Dieses Wachstum wie auch jede zukünftige Forderung nach mehr Stellen wird immer auch an ein Mehr an Verantwortung und Engagement geknüpft sein. Die Zeit sich auf den Status als kleines Orchideenfach zurückzuziehen ist vorbei. Zum einen bietet dieser inzwischen keinen Schutz mehr, zum anderen hat die Religionswissenschaft einen Vorschuss auf ihre gesellschaftliche Relevanz erhalten, den sie entschiedener einlösen muss. Deswegen sollte sie sich in gesellschaftlichen Fragen mehr zu Wort melden und in öffentliche Debatten stärker einmischen.

Das Fach muss mit mehr Geschlossenheit auftreten. Intern können verschiedene Fachverständnisse diskutiert werden, aber die Religionswissenschaft hat ein großes Problem, wenn nach außen nicht klar wird, wer sie vertritt. Damit verliert sie ihre Ansprechbarkeit und somit auch Relevanz für die Öffentlichkeit. Als positives Beispiel kann Göttingen hervorgehoben werden. Obwohl alle drei Professuren an verschiedenen Fakultäten angesiedelt sind, treten sie klar gemeinsam als Religionswissenschaft in Erscheinung und sind dadurch als solche identifizierbar. Ein geschlosseneres Auftreten dürfte auch die Position der Religionswissenschaft in Bezug auf die Profilbildung der Universitäten verbessern. Das Beispiel der Indologie in Halle zeigt aktuell, dass diese zu Gunsten der großen und auf Kosten der Kleinen Fächer erfolgt. Spezialfächer mit nur geringen Studierendenzahlen werden eingestellt - unabhängig davon wie traditionsreich und einmalig sie in Deutschland sind, wie aktuell sie sich ausrichten und wieviel Drittmittel sie einwerben. Die Frage ist, wie man diesem Umstand begegnet. Eine weitere Spezialisierung und damit auch Fragmentierung von Fächern und Disziplinen scheint jedenfalls die falsche Strategie zu sein. Vielmehr könnte man wieder mehr auf Entdifferenzierung setzen und den Zusammenschluss mit anderen Fächern suchen, vielleicht nach dem Vorbild der Departments an den Universitäten in den USA. Exklusivität und die Schaffung von Konkurrenzsituationen zu anderen, nicht selten größeren Fächern wird sich die Religionswissenschaft in Zukunft kaum leisten könnten. Dies hat sich als Strategie schon in den letzten Jahren nicht bewährt bzw. war nicht durchsetzbar. Eine stärkere Entdifferenzierung etwa von Religionswissenschaft und Theologien hat nicht stattgefunden. Sie wird wohl von Seiten der Theologien auch in den nächsten Jahren nicht erfolgen. Diese haben selbst ein Interesse daran, die 
religionswissenschaftliche Kompetenz in ihrem Fächerspektrum zu stärken, um sich darüber gesellschaftlich zu legitimieren und für Studierende attraktiv zu sein.

Die zentrale Kompetenz der Religionswissenschaft beruht dem Wissenschaftsrat zufolge in der Syntheseleistung religionsbezogener Wissensproduktion verschiedener Disziplinen. Fachgrenzen werden aber immer wieder als Kontravalenz formuliert: Jemand gehört entweder zur Religionswissenschaft oder zu einem anderen Fach, aber nicht beides zugleich. Dies führt zu Definitionen darüber, was die Religionswissenschaft als Fach genuin ausmacht im Unterschied $\mathrm{zu}$ anderen Fächern. Das Alleinstellungsmerkmal der Religionswissenschaft besteht aber weder in ihrem Gegenstand noch in ihren Methoden und Theorien, sondern vielmehr in einer zusätzlichen Kompetenz, dem Vergleich und der Synthese des von ihr selbst aber auch von anderen Disziplinen zum Gegenstandbereich Religion generierten Wissens. Daraus folgt, dass man in der Religionswissenschaft immer mindestens zweierlei ist: Religionswissenschaftler und Historiker, Religionswissenschaftlerin und Soziologin, Religionswissenschaftler und Islamwissenschaftler, Religionswissenschaftlerin und Indologin, Religionswissenschaftler und Theologe, Religionswissenschaftlerin und Ethnologin.

Religionswissenschaft stellt gewissermaßen die Schnittmenge aller religionsbezogen arbeitenden Fächer dar. Dies prädestiniert sie eigentlich dafür, eine leitende und integrative Funktion in der religionsbezogenen Wissensproduktion zu übernehmen. Das heißt, die Religionswissenschaft führt die verschiedenen religionsbezogen arbeitenden Disziplinen an und zusammen, sie tritt als Schirmherrin und Moderatorin auf. Dieser Rolle war sie lange Zeit als kleines Fach schlicht nicht gewachsen. Inzwischen ist die Religionswissenschaft aber an etlichen Standorten mit drei oder mehr Professuren vertreten. Beispiele wie das CERES in Bochum oder die KollegForschungsgruppe multiple secularities in Leipzig zeigen dabei, dass die Religionswissenschaft dort besonders erfolgreich ist, wo sie Führungsverantwortung mitübernimmt und als Initiatorin interdisziplinär angelegter Projekte und Strukturen auftritt. Diese Verantwortung und die damit verbundene Mehrarbeit muss man allerdings bereit sein zu übernehmen. Vielleicht wäre aber auch schon viel gewonnen, wenn in der Selbstthematisierung der Religionswissenschaft von Kontravalenz auf Konjunktion und Doppelzugehörigkeit umgestellt würde. Damit würde es attraktiver werden, sich zur Religionswissenschaft zu bekennen und innerhalb des Fachs $\mathrm{zu}$ bewegen und $\mathrm{zu}$ vernetzen. Muss man sich hingegen zwischen der Religionswissenschaft und einem anderen Fach entscheiden, dann fällt die Entscheidung schnell zu Ungunsten der Religionswissenschaft und für das andere, oft größere Fach aus. Kann man sich aber zugleich zu beiden Fächern bekennen, würde dies auch zu einer besseren Identifizierbarkeit der Religionswissenschaft führen. Es gäbe dann keine Verwirrung darüber, ob jemand zu ihr oder zu einem anderen Fach gehörte, sondern er bzw. sie gehört dann zu beiden. Hier böte sich das Instrument der Doppelprofessur an, wie es auch der Wissenschaftsrat eher am Rande vorgeschlagen hat. So könnten etwa Professuren, die bisher als Religionssoziologie nur in der Soziologie verankert sind, auch institutionell an die Religionswissenschaft angebunden werden.

$\mathrm{Zu}$ überlegen ist weiterhin, ob sich die Religionswissenschaft nicht viel stärker in der Lehramtsausbildung engagieren sollte. Hier ist sie bisher vor allem an den theologischen Fakultäten in der Ausbildung für den konfessionellen 
Religionsunterricht beteiligt. An philosophischen Fakultäten ist dies bei der Ausbildung von Ethiklehrern und Ethiklehrerinnen nicht der Fall, obwohl bei Durchsicht der Lehrpläne für Ethik ca. ein Drittel der Themen religionsbezogen ist. Grundsätzlich stellt sich die Frage, wenn religionswissenschaftliche Kompetenzen gesellschaftlich so relevant sind, dass sie einen integralen Bestandteil des Schulunterrichts bilden, und es an den Universitäten ein eigenes Fach Religionswissenschaft gibt, warum dann dieses Fach nicht oder nur als Hilfswissenschaft bei der Gestaltung des Unterrichtsfachs und der Lehramtsausbildung beteiligt ist. Mehr Engagement in der Lehramtsausbildung böte zwei strategische Vorteile: Dies würde verlässlich mehr Studierende für das Fach generieren und dessen gesellschaftlicher Relevanz entsprechend einen zentralen Beitrag im Bildungsbereich leisten. Natürlich würde das in vielen Bundesländern neue Modelle für den Religionsunterricht erfordern, die nur sehr schwer und langfristig durchzusetzen sind und für die sich die Religionswissenschaft Partner suchen müsste in den Religionsgemeinschaften, den Theologien und/oder der Philosophie. Es wäre sicherlich ein beschwerlicher Weg. Aber was passiert, wenn die Religionswissenschaft ihn nicht geht? Es steht zu befürchten, dass andere Akteure und Fächer das religionswissenschaftliche Kompetenzfeld stärker an sich ziehen und damit der Religionswissenschaft den Rang ablaufen werden. In einigen Bundesländern werden außerdem bereits alternative Modelle erprobt, von deren Erfahrungen profitiert werden kann - z.B. vom Model LER (Lebensgestaltung-Ethik-Religion) in Brandenburg.

Auch im Bereich der Masterstudiengänge könnte man umdenken, um die gesellschaftliche Relevanz der Religionswissenschaft zu erhöhen und mehr Studierende zu gewinnen. Wenn religionswissenschaftliche Kompetenzen eher als Zusatzkompetenz gefragt sind, dann könnten nicht-konsekutive Masterprogramme eine Option sein. Diese würden nicht auf einem religionswissenschaftlichen oder anderen geistes- und kulturwissenschaftlichen Bachelor aufbauen, sondern sich an Interessierte aus ganz verschiedenen Berufsgruppen richten. Für viele, deren politische, soziale oder wirtschaftliche Arbeit einen häufigen Kontakt mit Angehörigen verschiedener Religionen mit sich bringt, zwischen denen und einer nicht-religösen Umwelt vermittelt werden muss, kann eine Qualifikation in Religionswissenschaft eine wichtige Weiterbildung darstellen. Solche Master müssten berufsbegleitend studierbar sein, vielleicht sogar vorwiegend im eLearning. Hier könnte man von den Erfahrungen und der Digitalisierung durch die Pandemie profitieren.

Unter \#IchBinHanna ist jüngst auf Missstände im Bereich des wissenschaftlichen Nachwuchses aufmerksam gemacht worden. Einige der dort angesprochenen Probleme werden sich in der Religionswissenschaft in den nächsten zehn Jahren sogar noch verschärfen: Einem Mehr an Promovierten wird ein Weniger an neu zu besetzenden Professuren gegenüberstehen. Die Chancen, einmal auf einer wissenschaftlichen Dauerstelle zu landen, waren nie groß und sinken damit weiter erheblich. Die Frage, wie die Religionswissenschaft mit diesem Umstand umgehen soll, stellt sich mit zunehmender Dringlichkeit. 


\section{BIBLIOGRAPHY}

DVRW. N.d. »Religionswissenschfatliche Institute, Seminare und Lehrstühle im deutschsprachigen Raum« Letzter Zugriff: 19. Mai 2021. https://www.dvrw.uni-hannover.de/de/ religionswissenschaft/institute-seminar-lehrstuehle/

Hochschulkompass. N.d. »Erweiterte Studiengangssuche. Abschluss: Master. Fachsuche: Religionswissenschaft« Letzter Zugriff: 26. Mai 2021. https://www.hochschulkompass.de/ studium/studiengangsuche/erweiterte-studiengangsuche/search/1/studtyp/3.html?

tx_szhrksearch_pi1\%5Bfach\%5D=Religionswissenschaft\&tx_szhrksearch_pi1\%5Babschluss\%5D\%5B0\%5D=37

Kenngott, Eva und Englert, Rudolf und Knauth, Thorsten, Hrsg. 2015. Konfessionell - interreligiös religionskundlich. Unterrichtsmodelle in der Diskussion. Stuttgart: Kohlhammer.

Kleine Fächer. N.d. »Religionswissenschaft« Letzter Zugriff: 11. Mai 2021. https:// www.kleinefaecher.de/kartierung/kleine-faecher-von-a-z.html?

tx_dmdb_monitoring\%5BdisciplineTaxonomy\%5D=103\&cHash=5bc049c13b549a7728cd03bccab7a71d

Röther, Christian. 2017. »Gelehrtes Schweigen.« Deutschlandfunk 13. September 2017. Letzter Zugriff: 28. Mai 2021. https://www.deutschlandfunk.de/religionswissenschaft-gelehrtesschweigen.886.de.html?dram:article_id=395657

Schröder, Stefan. 2020. »Umstrittene Säkularität. Säkularer Religionsunterricht an öffentlichen Schulen.« Zeitschrift für Religionswissenschaft 28(2): 314-335.

Statistisches Bundesamt. 2010/11. 2011. Bildung und Kultur. Studierende an Hochschulen. Wintersemester 2010/11. Letzter Zugriff: 21. Mai 2021. https://www.statistischebibliothek.de/ mir/receive/DEHeft_mods_00005831

Statistisches Bundesamt. 2015/16. 2016. Bildung und Kultur. Studierende an Hochschulen. Wintersemester 2015/16. Letzter Zugriff: 21. Mai 2021 https://www.statistischebibliothek.de/ mir/receive/DEHeft_mods_00056537

Statistisches Bundesamt. 2019/20. 2020. Bildung und Kultur. Studierende an Hochschulen. Wintersemester 2019/20. Letzter Zugriff: 21. Mai 2021 https://www.statistischebibliothek.de/ mir/receive/DEHeft_mods_00131465

Statistisches Bundesamt. 2020. Bildung und Kultur. Statistik der Promovierenden 2019. Letzter Zugriff: 22. Mai 2021. https://www.destatis.de/DE/Themen/Gesellschaft-Umwelt/Bildung-ForschungKultur/Hochschulen/Publikationen/Downloads-Hochschulen/ promovierendenstatistik-5213501197004.pdf?__blob=publicationFile

Uni Halle. N.d. »Prof. Dr. Daniel Cyranka« Letzter Zugriff: 19. Mai 2021. https:// www.theologie.uni-halle.de/rw/cyranka/

Uni Hamburg. N.d. »Institut für Missions-, Ökumene- und Religionswissenschaft« Letzter Zugriff: 11. Juni 2021. https://www.theologie.uni-hamburg.de/einrichtungen/institute/moer.html

Uni Hannover. N.d. »Geschichte des Instituts für Religionswissenschaft« Letzter Zugriff: 11. Juni 2021. https://www.irw.uni-hannover.de/de/institut/geschichte-des-instituts/

Uni Heidelberg. N.d. »Mitarbeitende« Letzter Zugriff: 11. Juni 2021. https:// religionswissenschaft.zegk.uni-heidelberg.de/mitarbeiter/index.html 
Uni Rostock. N.d. »em. Prof. Dr. Klaus Hock« Letzter Zugriff: 21. Mai 2021 https:// www.theologie.uni-rostock.de/fachgebiete/religionswissenschaft-und-interkulturelle-theologie/ prof-dr-klaus-hock/

Wissenschaftsrat. 2010. Empfehlungen zur Weiterentwicklung von Theologie und religionsbezogenen Wissenschaften an deutschen Hochschulen. Letzter Zugriff: 26. März 2021. https:// www.wissenschaftsrat.de/download/archiv/9678-10.pdf?__blob=publicationFile\&v=1

\section{NOTES}

1. Allerdings mit etwas eigenartigen Zeitangaben: Zwei der Professuren seien erst 2017 dazugekommen. Aus den Lebensläufen der beiden Innehabenden geht aber hervor, dass beide Professuren zunächst als Juniorprofessuren besetzt und 2015 in dauerhafte Professuren umgewandelt wurden.

2. Eigene Zählung von Professuren (keine apl. Prof.) mit einer religionswissenschaftlichen Denomination an einem der 28 vom Wissenschaftsrat 2009 gelisteten Standorte. Die Informationen stammen von den jeweiligen Internetauftritten und CVs der Professuren. Nicht alle machen Angaben zum Zeitpunkt der Berufung, beim CERES in Bochum etwa wird oft auf diese Angabe verzichtet. Ich erhebe mit dieser Zählung keinen Anspruch auf Vollständigkeit oder Ausschließlichkeit. Auf eine detaillierte Aufschlüsselung der Professuren wird deshalb verzichtet. 3. Beachtet wurden die Jahrgänge 1954-1962. Eigene Zählung gemäß den Internetauftritten und CVs der Professuren. Orientiert an den Standorten der Zählung des Wissenschaftsrats. Auch hier erhebe ich wieder keinen Anspruch auf Vollständigkeit.

\section{ABSTRACTS}

Dieser Artikel zeichnet anhand der religionswissenschaftlichen Professuren die Entwicklung des Fachs in Deutschland innerhalb der letzten zehn Jahre nach und wagt davon ausgehend eine Prognose für die nächsten zehn. Der Wissenschaftsrat hatte sich 2010 ausgehend von einer sog. Rückkehr der Religion und der damit verbundenen hohen gesellschaftlichen Relevanz des Fachs dafür ausgesprochen, die Religionswissenschaft durch mehr Professuren zu stärken. Dieser Empfehlung wurde an vielen Orten gefolgt. Das Wachstum an Professuren korrespondiert allerdings nicht mit einer in gleicher Weise gesteigerten Präsenz der Religionswissenschaft in außeruniversitären Diskursen. Hier werden andere Fächer wie die Theologien, Geschichte und Soziologie stärker gehört. Angesichts tendenziell sinkender Studierendenzahlen und der Schließung anderer Kleiner Fächer stellt sich deshalb die dringende Frage, wie sich die Religionswissenschaft strategisch positionieren sollte.

This article depicts the development of the scientific study of religion in Germany within the last ten years on the basis of professorships and ventures a prognosis for the next ten years. In 2010, ensuing from a so-called return of religion and the subsequent high social relevance of the discipline, the Wissenschaftsrat (academic council) advocated reinforcing the study of religion through more professorships. This recommendation has been pursued throughout many places. However, the growth of professorships does not correspond to an equally increased presence of 
the study of religion in non-academic discourses. Here, other disciplines such as the theologies, history, and sociology are more likely to be heard. Given the downward trend of student numbers as well as the closures of other small disciplines, the urgent question arises as to how the study of religion should position itself strategically as a discipline and how it shall fulfill its social relevance in the future.

\section{AUTHOR}

\section{ULRIKE KOLLODZEISKI}

Ulrike Kollodzeiski hat Religionswissenschaft, Jüdische Studien und Geschichte der Frühen Neuzeit an den Universitäten Potsdam und Leiden studiert. Sie hat an der Goethe Universität Frankfurt a.M. in Religionswissenschaft mit der Arbeit »Die Ordnung der Religionen. Die Vermittlung von Orient und Okzident im Reisebericht Viaggi von Pietro Della Valle (1586-1652)« promoviert. Seit 2019 arbeitet sie im Institut für Jüdische Studien und Religionswissenschaft an der Universität Potsdam und forscht zum Thema Speiseregeln im antiken Christentum.

Kontakt: kollodzeiski@uni-potsdam.de 